\title{
Collisional decoherence during writing and reading quantum states
}

\author{
Stephanie Manz, ${ }^{1,2}$ Thomas Fernholz, ${ }^{1,3}$ Jörg Schmiedmayer, ${ }^{1,2}$ and Jian-Wei Pan ${ }^{1}$ \\ ${ }^{1}$ Physikalisches Institut, Universität Heidelberg, 69120 Heidelberg, Germany \\ ${ }^{2}$ Atominstitut Österreichischer Universitäten, TU-Wien, A-1020 Vienna, Austria \\ ${ }^{3}$ Van der Waals-Zeeman Institute, University of Amsterdam, 1018 XE Amsterdam, The Netherlands
}

(Dated: September 9, 2018)

\begin{abstract}
Collisions, even though they do not limit the lifetime of quantum information stored in ground state hyperfine coherences, they may severely limit the fidelity for quantum memory when they happen during the write and read process. This imposes restrictions on the implementation of Raman type quantum processes in thermal vapor cells and their performance as a quantum memory. We study the effect of these collisions in our experiment.
\end{abstract}

PACS numbers: 32.80.Qk, 03.67.Hk, 03.65.Yz

In recent years, significant experimental advances have been achieved in the field of quantum communication (QC) 1, 2]. However, photon loss and detector noise limit direct QC to moderate distances (up to $100 \mathrm{~km}$ in quantum cryptography). In 2001, Duan, Lukin, Cirac and Zoller (DLCZ) proposed a practical quantum repeater 3, 4] based on writing and reading single excitations in atomic ensembles using Raman type processes. A joint projective measurement of individual photons emitted from two separated atomic ensembles leads to qubittype entanglement of collective excitations in both ensembles, which combined with entanglement swapping [5] and entanglement purification [6] allows to create entanglement over (arbitrary) long distances. Essential to the scalability of the DLCZ scheme are the long lived collective excitations, which represent a quantum memory. Without such quantum memory, the overhead scales exponentially with the channel length.

Although entanglement swapping [7] and entanglement purification [8] have been experimentally demonstrated with linear optics, it is difficult to achieve the high fidelity quantum memory. Significant experimental advances have been made to implement the DLCZ-scheme 3n both with ultra-cold atomic clouds 9, 10, 11, 12, 13, 14] and hot vapors in buffer gas cells [15, 16, 17]. While ultracold ensembles require substantial technological effort, vapor cells provide comparatively easy experimental access. Moreover atomic clock experiments have shown that with the correct coating of cell walls and the use of buffer gas the ground state coherence can be preserved for up to $>10^{8}$ collisions [18] leading to very narrow line widths.

In this paper, we demonstrate however that the mapping process between light fields and atomic coherence is strongly influenced by collisions. This severely limits the fidelity of the DLCZ-scheme and a quantum memory in hot atomic ensembles.

The mapping process between light fields and atomic coherence is essential to the DLCZ scheme [3]. Single excitations are written in an atomic ensemble Raman transitions in a three level $\lambda$-configuration: (ground states

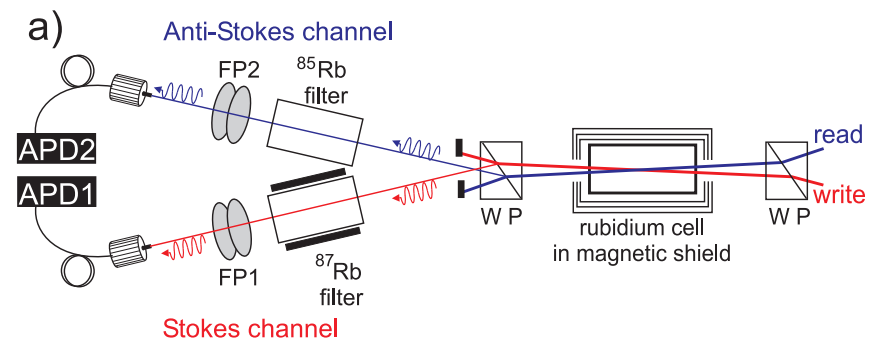

b)

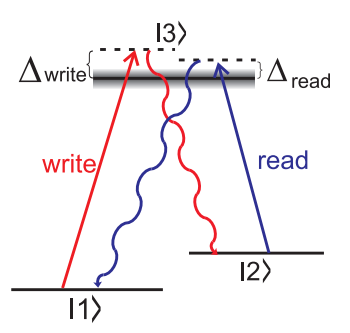

c)

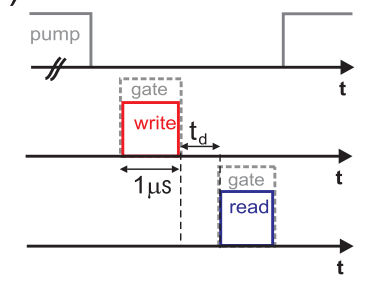

FIG. 1: (color online) a) Experimental setup: Two control lasers (write and read) are combined at a Wollastone prism (WP) and intersect inside a magnetically shielded ${ }^{87} \mathrm{Rb}$ vapor cell with Neon buffer gas. The weak signal fields (Stokes and Anti-Stokes) are separated from the control beams at a second polarizer. After spectral filtering, the signal photons are detected by fiber coupled avanlanche photo diode (APD). b) Simplified level scheme: A lambda system is used to map ground state coherences to the signal fields via two detuned control beams. c) Laser pulse timing for spontaneous creation of ground state coherence and delayed read-out.

$|1\rangle,|2\rangle$, excited state $|3\rangle)$. First, the atoms are optically pumped to $|1\rangle$. Then a write-laser, detuned by $\Delta_{\text {write }}$ from the $|1\rangle \rightarrow|3\rangle$ transition induces a spontaneous Raman process. Upon detection of a Stokes photon the atoms are projected to a long-lived collective state (the quantum memory), corresponding to a spin-wave excitation. This process can be inverted with a read laser detuned $\left(\Delta_{\text {read }}\right)$ from the $|2\rangle \rightarrow|3\rangle$ transition, which converts the spin excitation back to Anti-Stokes photons.

In our experimental implementation (Fig. 1) we use hot ${ }^{87} \mathrm{Rb}$ vapor as the atomic medium. The levels form- 
ing the $\lambda$-system are given by the $5^{2} S_{1 / 2}, F=1$ and $5^{2} S_{1 / 2}, F=2$ ground states (corresponding to $|1\rangle$ and $|2\rangle)$, and the $5^{2} P_{1 / 2}$ manifold comprised in the excited state $|3\rangle$. The write and read processes are controlled by two nearly co-propagating laser beams with orthogonal linear polarizations $\left(P_{\text {write }} \approx 1 \mathrm{~mW}\right.$ and $\left.P_{\text {read }} \approx 5 \mathrm{~mW}\right)$ focussed to $\approx 300 \mu \mathrm{m}$ diameter intersecting inside the cell at a small angle $\alpha \approx 5$ mrad. For linear polarizations, the signal beams are mainly generated with polarizations orthogonal to their respective control beams [19], and can be separated at a crystal polarizer (Wollaston prism). The signals are detected with electronically gated, fibre coupled avalanche photon detectors (PerkinElmer, SPCM-AQR series). This set-up resembles the one desribed in 15].

To obtain high enough optical depth, the Rb is heated to $\mathrm{T}=60^{\circ} \mathrm{C}$, and $p_{N e} \approx 7$ torr of Neon buffer gas keeps the hot atoms long enough in the interaction region. All is confined in a $4 \mathrm{~cm}$ long glass-cell inside a three layer $\mu$-metal shield to reduce magnetic stray fields.

In order to achieve a high enough signal-to-noise ratio, we employ various filter techniques to suppress leakage from the strong write (read) beams. Before entering the atomic ensemble FP filter cavities reduce the spectrally broad amplified spontaneous emission (ASE) from the write (read) lasers. These resonators also provide spatially clean beam profiles. Due to the limited extinction ratio of the crystal polarizer $\left(\approx 10^{-5}\right)$, the strong control beams leak into the respective signal channels, and further filtering is essential. The write (read) lasers are blue detuned from the $F=1(2) \rightarrow F^{\prime}=2$ transitions, $\Delta$ ranging between $0.5 \mathrm{GHz}$ and $1.5 \mathrm{GHz}$. The read channel is filtered by a hot, isotopically enriched ${ }^{85} \mathrm{Rb}$ vapor cell, which is opaque for the control (read) but transparent for the signal (Anti-Stokes) frequency. For the write channel, we use an optically pumped ${ }^{87} \mathrm{Rb}$ vapor cell that blocks residual write light in the Stokes signal. The absorption of the filter cell is broadened by a magnetic field of $\approx 0.1$ Tesla using permanent magnets, allowing for larger detunings of the write laser.

In order to further examine the generated quantum fields in detail, we employ additional Fabry-Perot etalons (free spectral range $\mathrm{FSR}=5 \mathrm{GHz}$ with FWHM-linewidth of $480 \mathrm{MHz}$ ), which allow us to perform frequency resolving scans of the signal channels. Fig. 2 shows a scan of the Stokes channel, while the Fabry-Perot interferometer in the Anti-Stokes channel was tuned to maximum transmission (0.8 counts per pulse). Each data point represents 4000 experimental cycles, which were performed at a repetition rate of $10 \mathrm{kHz}$. The bottom left of Fig. 2 shows the intensity spectrum, represented by the mean photon number per pulse. As a frequency reference, we included a small fraction of the write light by reducing the temperature of the filter cell, labelled as (L) in fig. 2. The write light was blue detuned from the $F=1 \rightarrow F^{\prime}=2$ transition by $800 \mathrm{MHz}$. We find Stokes light at a relative

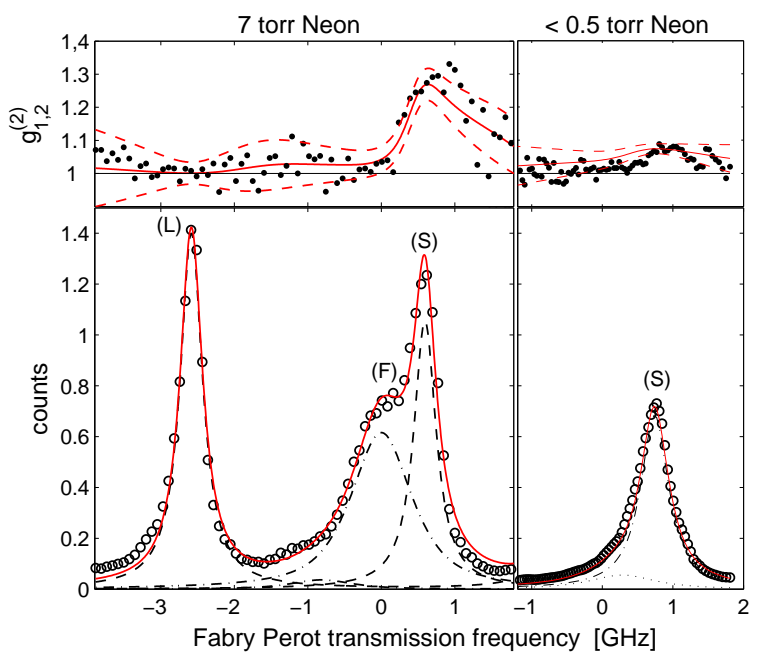

FIG. 2: (color online)(left) Frequency resolved scan of the Stokes channel with imperfectly filtered write light as a frequency reference. The length of a Fabry-Perot interferometer $(\mathrm{FP} 1, \mathrm{FSR}=5 \mathrm{GHz})$ was scanned while the signal intensity (bottom) and its cross correlation to the Anti-Stokes channel (top) were recorded. The model (solid red line) includes the coherent (S) and incoherent (F) contributions to the Stokes signal, and the write laser light (L) that appears again two FSR away from its original frequency. The data (black dots) show that only the coherent Stokes contribution is correlated to the Anti-Stokes signal. Statistical uncertainties in the measured cross correlation were calculated according to the finite number of experimental cycles. The red dashed lines indicate confidence bands for one sigma deviation. (right) Corresponding plots for the mean of four measurements with reduced buffer gas pressure below 0.5 torr.

red detuning corresponding to the hyperfine splitting frequency of ${ }^{87} \mathrm{Rb}(6.83 \mathrm{GHz})$. This frequency is amplified by the medium, and the intensity of this signal can be controlled by the length of the write pulse and its detuning from the D1 transition frequency.

The striking observation is that for a vapor cell with buffer gas $(\sim 7$ torr Neon), an additional contribution to the signal is present, close to the Stokes frequency. The intensity of this signal is independent of the pulse length, and it only becomes significant at the single (few) photon level, where amplification of the Stokes light is negligible. Scans of the retrieve channel revealed a similar signal close to the Anti-Stokes frequency.

We attribute the additional signal to incoherent Raman scattering of the write (read) light, caused by collisional perturbation of the excited state during the write (read) process. This process is included in mechanisms described in [20] and is similar to collision induced fluorescence reported in 21] for the strongly stimulated regime. Its frequency should be resonant with the atomic transition. For a more quantitative comparison, we model the incoherent signal using two Voigt lines with 


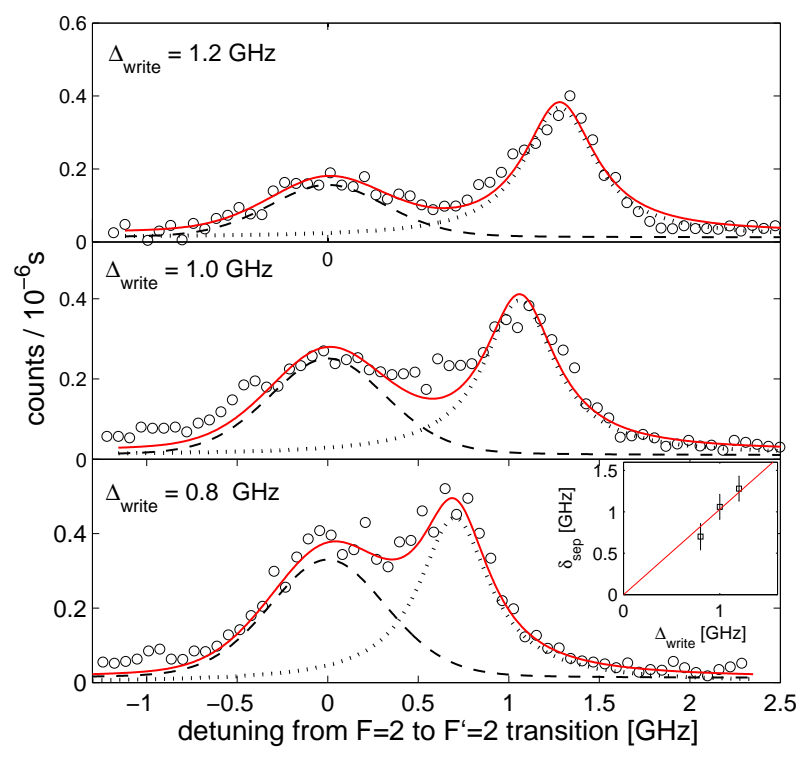

FIG. 3: (color online) Coherent and incoherent contributions to the Stokes signal for various write detunings. The three upper plots show frequency scans of the Stokes channel, using a Fabry-Perot resonator with a linewidth of $480 \mathrm{MHz}$ and an FSR of $5 \mathrm{GHz}$. The solid line is a fitted model, including a sharp Stokes peak (dotted) and a broader fluorescence peak (dashed) convoluted with the Fabry-Perot transmission function. The width of the fluorescence is assumed to be given by the Doppler profile according to the atomic temperature. The inset displays the approximate equality of peak separation and write detuning.

a separation of $812 \mathrm{MHz}$, given by the hyperfine splitting of the excited state. The FWHM-line widths are fixed to $480 \mathrm{MHz}$ for the Gaussian part, given by the Doppler broadening of Rubidium at a temperature of $60^{\circ} \mathrm{C}$, and the Fabry-Perot line width for the Lorentzian part. We find a significant contribution from the upper excited level only. To support this interpretation we performed similar measurements at different detunings (Fig. 3). The frequency difference between the two components equals the write detuning from the $F=2 \rightarrow F^{\prime}=2$ transition.

With the frequency resolved detection we can investigate the coherent nature of the written collective excitation. The upper part of Fig. 2 shows the measured cross correlation $g_{1,2}^{(2)}=\left\langle n_{1} n_{2}\right\rangle /\left\langle n_{1}\right\rangle\left\langle n_{2}\right\rangle$ between the two signal beams. The solid line gives the prediction under the assumption that collisions during the write process lead to a completely dephased excitation.

While the observed cross correlation is independent of the detection efficiencies, it depends on the signalto-noise ratios $\chi_{1}$ and $\chi_{2}$ in the two channels as $g_{1,2}^{(2)}-$ $1=\left(g_{S, A S}^{(2)}-1\right)\left(1+1 / \chi_{1}\right)^{-1}\left(1+1 / \chi_{2}\right)^{-1}$, where $g_{S, A S}^{(2)}$ is the cross correlation between the noise-free signals. Our data can be described by a single free parameter
$\left(g_{S, A S}^{(2)}-1\right)\left(1+1 / \chi_{2}\right)^{-1}=0.35$. The good agreement of our data with this model shows that only upon detection of coherently scattered Stokes light, the ground state coherence can be converted into correlated AntiStokes light during the read pulse, and collisions during the read and write pulse lead to destruction of the quantum excitation.

To support our assumptions, frequency resolving scans have also been taken with strongly reduced buffer gas pressure $(\sim 0.2$ torr $)$. In this experiment the generation of coherent Stokes light was enhanced, and we lowered the temperature of the $\mathrm{Rb}$ vapor ( $\mathrm{Rb}$ density) to obtain similar count rates in the Stokes channel for the same write parameters. At low buffer gas pressure, no significant incoherent contributions are apparent. However, the detection of cross correlation was hampered by the fast atomic diffusion. Atoms leave the interaction region too quickly, resulting in a strongly reduced retrieve efficiency and a low signal-to-noise ratio in the Anti-Stokes channel.

The observed decoherence processes during write and read might be related to a recent theoretical work by Childress et al. [22]. They predict the appearance of incoherently scattered light for color centers in diamond. They model the perturbations of the excited level as a time dependent detuning with white noise characteristics under the assumption that the perturbing mechanism is slow compared to the optical frequency but faster than the radiative lifetime of the excited state. Their model predicts coherent and incoherent signal contributions with a relative frequency difference equal to the detuning of the driving laser from the atomic transition. The relative weights are given by $\gamma: \Gamma$, where $\Gamma$ is the fluctuation amplitude of the excited state detuning and $\gamma$ its radiative decay rate.

The model qualitatively also describes our findings, especially the frequency difference between the coherent and incoherent part of the spectrum (Fig. 3). In our case the perturbations are given by the collisions, which behave like delta function perturbations. From a broadening coefficient [23] of $\gamma_{c} \approx(7 \pm 2) M H z /$ torr [24] we estimate a collision rate for atoms undergoing a Raman transition of $\gamma_{c} \approx 49 \mathrm{MHz}$ at 7 torr Neon gas pressure. For these parameters collisions happen on a similar timescale compared to the D1 decay rate of $\gamma=36 \times 10^{-6} s^{-1}$. For very low Stokes amplification we observe the relative weights between the coherent and incoherent part to be roughly equal. In the strong collision limit their ratio should be given by the branching ratio $\gamma_{R} \approx \gamma_{c}: \gamma$ of excited state decay with and without intermediate collisions. An accurate description would require an elaborate model that includes a detailed description of the coherence in the Raman process in this collisional regime, inhomogeneous doppler broadening and velocity redistribution induced by the collisions, effects of collective enhancement in the atomic ensemble and the substructure 
of the excited level. Our experiments have shown, that disturbances during writing and reading collective excitations are very important for the performance of quantum processes. Whereas one usually selects very stable protected states to store the quantum information, the states involved during reading and writing can be very fragile. If one uses the hyperfine ground states of alkali atoms, the coherence in the nuclear spin states is shielded from collisions by the electrons of the atom, as given by dark state coherence or under EIT conditions. Using special coated cells and noble buffer gas the atoms can endure many $\left(>10^{8}\right)$ collisions before loosing hyperfine coherence. The Raman transition based write and read processes of a quantum memory involves electronically excited states, and a single collision can destroy the coherence, and (quantum) information is lost.

These write and read errors can be very severe in quantum applications. For some, like the quantum repeater they can be filtered out by selecting only photons on the unperturbed Raman line. But even then they lead to increased overhead, since the fidelity of a single step is reduced. To keep the polynomial scaling one must make sure that the overhead does not lead to exhaustion of the memory coherence time. For a quantum memory the decoherence in the write and read processes are more severe and directly reduce fidelity.

Consequently, to achieve high fidelity quantum operations these incoherent processes need to be suppressed. For thermal ensembles this requires reducing the probability of collisions during the read and write process, which means reducing the buffer gas pressure. This leads to a greatly increased diffusion length. To keep a good retrieve efficiency and long storage time one must keep the atoms in a tight overlap with read and write beams. For an increased diffusion length this can be done by a larger beam diameter. This makes the filtering of unwanted light much harder, since the required total power has to be increased. A better way might be to put the atoms in a hollow fiber 25], which can also be used to guide the read and write light. The atoms are then kept in the laser beam by collisions with the walls, and one can refrain from using buffer gas at all. For a thermal velocity of $\sim 300 \mathrm{~m} / \mathrm{s}$ the typical atom wall collision rate will be $\sim 3 \times 10^{6}$ in a $100 \mu m$ diameter hole. Again collisions between the wall and atoms in the excited state will lead to decoherence, and should be avoided. One can achieve this by forcing the light field to be zero at the wall. Working far off resonance, the atoms will evolve adiabatically to the ground state when being excited by the light field in approaching the wall. This would require the fiber hole to be coated by a mirror which also protects the stored atomic spin. A different approach could be to design the guided light field by a photonic band gap structure, keeping it minimal close to the walls.

In conclusion we point out that collisions, even though they might not limit the life time of a stored ground state hyperfine coherence, dark states or EIT, they may severely limit the fidelity for this type of quantum memory when they happen during the write and read process. These collisions, be it with the buffer gas atoms or cell walls are required to keep the atoms in the write / read volume when using thermal vapor cells. Cold atoms have a great advantage because they allow wall free confinement, and the collision processes happen at timescales many orders of magnitude slower than the read and write processes.

This work was supported by the Deutsche Forschungsgemeinschaft E. Noether programm contract number PA938 and European Union, Integrated Project FET/QIPC "SCALA". We thank Igor Mazets for helpful discussions.

[1] N. Gisin, G. Ribordy, W. Tittel, and H. Zbinden, Rev. Mod. Phys. 74, 145 (2002).

[2] A. Zeilinger, G. Weihs, T. Jennewein, and M. Aspelmeyer, Nature (London) 433, 230 (2005).

[3] L.-M. Duan, M. D. Lukin, J. I. Cirac, and P. Zoller, Nature (London) 414, 413 (2001).

[4] H.-J. Briegel, W. Duer, J. I. Cirac, and P. Zoller, Phys. Rev. Lett. 81, 5932 (1998).

[5] M. Zukowski, A. Zeilinger, M. A. Horne, and A. Ekert, Phys. Rev. Lett. 71, 4287 (1993).

[6] C. H. Bennett, G. Brassard, S. Popescu, B. Schumacher, J. A. Smolin, and W. K. Wootters, Phys. Rev. Lett. 76, 722 (1996).

[7] J.-W. Pan, D. Bouwmeester, H. Weinfurter, and A. Zeilinger, Phys. Rev. Lett. 80, 3891 (1998).

[8] J.-W. Pan, S. Gasparoni, R. Ursin, G. Weihs, and A. Zeilinger, Nature (London) 423, 417 (2003).

[9] A. Kuzmich, W. P. Bowen, A. D. Boozer, A. Boca, C. W. Chou, L.-M. Duan, and H. J. Kimble, Nature (London) 423, 731 (2003).

[10] D. N. Matsukevich and A. Kuzmich, Science 306, 663 (2004).

[11] D. N. Matsukevich, T. Chanelière, S. D. Jenkins, S.-Y. Lan, T. A. B. Kennedy, and A. Kuzmich, Phys. Rev. Lett. 97, 013601 (2006).

[12] S. Chen, Y.-A. Chen, T. Strassel, Z.-S. Yuan, B. Zhao, J. Schmiedmayer, and J.-W. Pan, Phys. Rev. Lett. 97, 173004 (2006).

[13] C. W. Chou, H. de Riedmatten, D. Felinto, S. V. Polyakov, S. J. van Enk, and H. J. Kimble, Nature (London) 438, 828 (2005).

[14] T. Chanelière, D. N. Matsukevich, S. D. Jenkins, S.-Y. Lan, T. A. B. Kennedy, and A. Kuzmich, Nature (London) 438, 833 (2005).

[15] C. H. van der Wal, M. D. Eisaman, A. André, R. L. Walsworth, D. F. Phillips, A. S. Zibrov, and M. D. Lukin, Science 301, 196 (2003).

[16] A. André, M. D. Eisaman, R. L. Walsworth, A. S. Zibrov, and M. D. Lukin, J. Phys. B 38, S589 (2005).

[17] M. D. Eisaman, A. André, F. Massou, M. Fleischhauer, A. S. Zibrov, and M. D. Lukin, Nature (London) 438, 837 (2005). 
[18] W. Happer, Rev. Mod. Phys 44, 169 (1974).

[19] R. Wynands, A. Nagel, S. Brandt, D. Meschede, and A. Weis, Phys. Rev. A 58, 196 (1998).

[20] Y. R. Shen, Phys. Rev. B 9, 622 (1974); D. L. Rousseau, G. D. Patterson, P. F. Williams, Phys. Rev. Lett. 34, 1306 (1975).

[21] M. G. Raymer and J. L. Carlsten, Phys. Rev. Lett. 39, 1326 (1977).

[22] L. I. Childress, J. M. Taylor, A. S. Sørensen, and M. D. Lukin, Phys. Rev. A 72, 052330 (2005).

[23] We have to consider the collisional broadening and not the ground-state collision rate. They differ due to the different electrical polarizability for ground and excited states. We could not directly measure the collisional broadening in the measurement cell at the operating temperature.

[24] S. L. Izotova, A. I. Kantserov, and M. S. Frish, Opt. Spectrosc. 51, 107 (1981).

[25] F. Benabid, G. Antonopoulos, J. C. Knight, P. St. J. Russel, Phys. Rev. Lett. 95, 213903 (2005). 